\title{
Discussion on the Development of Anorectal Discology in Chinese Medicine at the Stage of Internet
}

\author{
Zongyue Gao ${ }^{1,2, a}$, Ning Zhang ${ }^{1,2, b}$, Zuowu Xi ${ }^{1,2, c}$ and Xiaoli Zhou ${ }^{3, d^{*}}$ \\ ${ }^{1}$ Henan Province Hospital of Traditional Chinese Medicine, 450002.Zhengzhou, 450000, Henan, P. \\ R. China \\ ${ }^{2}$ The Second Affiliated Hospital of Henan University of Traditional Chinese Medicine, 450000, \\ Henan, P. R. China \\ ${ }^{3}$ The Third Affiliated Hospital of Henan University of Traditional Chinese Medicine, 450000, Henan, \\ P. R. China \\ ahnszyygzy@126.com, bzhangninghntcm@163.com, \\ cxizuowuhntcm@163.com, 'zhouxiaolihntcm@163.com \\ *hnszyygzy@126.com
}

\section{Keywords: Anorectal Discology; Chinese Medicine; Internet; Network system; Development}

\begin{abstract}
The Internet is an important platform for academic exchange, business study, external publicity, display image and information sharing in Chinese anorectal discipline. In this paper, through the use of Internet technology, to explain the anorectal professional website construction should have the function and need to grasp the elements, focusing on the construction of anorectal industry characteristics of the site, integration of anorectal network resources, the formation of the Chinese anorectal professional network system, in order to promote the development of anorectal, To speed up the anorectal discipline in the pace of information technology for many patients with anorectal disease to provide convenient and professional services for the anorectal clinicians to provide an exchange of academic platform, thus promoting the development of anorectal discipline.

The Internet is a highly efficient and convenient, low-cost, global communications tool. With the Internet in the field of medical and health applications, many aspects of the health care industry has produced many new ideas, new methods, new means and new ways to become anorectal field of external publicity, display the image, an important platform for the exchange of information The Integration of related anorectal information, focus on the anorectal details, to all patients with anorectal disease to provide comprehensive services; patients on the Internet to find the required anorectal information, through online consultation, access to authoritative experts to answer; Medicine, an accurate understanding of the various anorectal hospital, anorectal experts and professional expertise and good at treating disease, easy to seek medical treatment; this site will provide free anorectal health knowledge, popularization of the masses of health education; website for the majority of anorectal health care workers To provide communication and interactive learning platform, can effectively improve the anorectal community information sharing and academic exchange level. ${ }^{[1]}$
\end{abstract}

\section{Present Anorectal Development Status Quo}

Information Asymmetry between Doctors and Patients. Anorectal disease is a common clinical disease, high incidence. Speed up the application of the Internet in clinical work, especially at this stage in the anorectal application research is the current anorectal field is facing a very important work. A total of 155 units were surveyed by the National Association of Integrated Traditional Chinese and Western Medicine. The total prevalence of anal rectal disease was 59.1\%. The patients with hemorrhoids accounted for $87.25 \%$ of patients with anorectal disease, the number of $46.3 \%{ }^{[2]}$. However, due to the particularity of the pathogenesis of anorectal disease, but not the most fatal disease, in the past most people are due to feelings or because of health and health concepts and conditions do not want to go to regular hospital treatment, resulting in delay the best treatment 
opportunity. With the rapid development of modern science, people's understanding of anorectal disease is constantly injected into the new content, anal mat theory, PPH technology, TST technology and other new theories, new technologies continue to emerge. However, due to long-term anorectal can not get the attention, the country's anorectal discipline from the department to the technical level of the specialist are uneven, the development of lagging behind, lack of positive publicity, resulting in the majority of patients with anorectal disease can not be successfully obtained The information required is blind in the selection of specialist hospitals or physicians ${ }^{[3]}$. And a very small number of for-profit private hospitals are taking this opportunity to use overwhelming advertising to mislead patients, resulting in a great waste of health economics, and delayed the best period of treatment of patients.

Knowledge Update Requires the Specialist to Constantly Adjust the Knowledge Structure. At present, there are still a lot of problems in the field of anorectal need to be further studied. Such as the pathogenesis of hemorrhoids has not yet elucidated, many treatment methods, the efficacy of different evaluation; colorectal cancer incidence gradually increased, how to further reveal the pathogenesis of colorectal cancer, looking for more effective prevention, treatment and improve the patient's 5 Chronic obstructive constipation has gradually become an important discussion of anorectal surgery, although constipation is only a functional disease, but known as "benign cancer," said the patient's physical and psychological aspects of a huge impact, And the current academic community on the basis of constipation, clinical research are in a state of stagnation. To solve the problems in the field of anorectal, better service for patients, relying solely on an expert or a hospital academic strength is not enough, which requires the entire anorectal community to work together, need specialists to constantly update the knowledge structure, based on this, The urgent need for an information sharing, exchange platform to promote the continuous improvement of the academic level of specialist ${ }^{[4]}$.

\section{The Status of Patients Demand}

Anorectal site of the surgical treatment and characteristics of other surgical different, in addition to the special site of the disease, the local physiology and anatomy in the human body is more specific, is the body's physical, psychological and functional three sensitive areas. With the economic development and improvement of people's living standards, changes in health concepts, people on the quality of life and health standards put forward higher requirements ${ }^{[5]}$. Anorectal disease patients in the pursuit of treatment not only the pursuit of elimination or alleviate the symptoms, but also requires anorectal sensation, recognition of the complete protection of the function, but also on the appearance of the anus made almost harsh requirements. Patients often need to obtain a comprehensive understanding of the disease, whether it is for physical or psychological needs, are hoping to get the correct answer to the authority of the experts. The Internet is the best platform for all information and information sharing.

\section{The Role of Website Construction in the Development of Anorectal}

Site Constructions is the Development of Anorectal Itself. the development of information technology is the inevitable trend of social development, anorectal professional website construction is an important component of anorectal information construction, and its importance is more and more obvious. On the one hand, Internet users through an anorectal professional website query, consult their own information needed to facilitate timely medical treatment; the other hand, through this platform to the domestic and international publicity, display anorectal discipline image and performance, the subject of the latest developments, clinical new technology , Scientific research, education and teaching, science and health knowledge and other information provided to the community, so as to serve the community ${ }^{[6]}$. Especially in the construction of professional industry website, in particular, is very important.

Website Constructions is to Establish Anorectal Discipline Brand Needs. In the modern market economy tide, the anorectal disciplines also need the public to understand the cognitive; in 
the current increasingly competitive medical market, a good brand will be one of the lives of a discipline development ${ }^{[7]}$. Internet to the anorectal discipline is connected to the world, large amount of information, real-time strong, low-cost advertising media, more convenient than traditional media.

Construction of Anorectal Professional Website is Anorectal Specialty Professional Needs. Anorectal site of the surgical treatment and characteristics of other surgical different, in addition to the special site of the disease, the local physiology and anatomy in the human body is more specific, is the body's physical, psychological and functional three sensitive areas. With the economic development and improvement of people's living standards, changes in health concepts, people on the quality of life and health standards put forward higher requirements ${ }^{[8]}$. Anorectal disease patients in the pursuit of treatment not only the pursuit of elimination or alleviate the symptoms, but also requires anorectal sensation, recognition of the complete protection of the function, but also on the appearance of the anus made almost harsh requirements. Patients often need to gain a comprehensive understanding of the disease, whether it is out of life

Rational or psychological needs, are hoping to get the right authority of the experts and the Internet is the best platform for all information and information sharing.

\section{Internet Technologies is Mature}

With the development of network information technology and the development trend of technical standards at home and abroad, the technology of website construction has experienced from the htm plain text website in 1999, the ASP language editing in 2003, and the present the JAVA development platform to achieve a great enrich and meet the needs of the masses at this stage. Web site with large capacity, multimedia, easy to retrieve, real-time, interactive, no geographical limitations and so on ${ }^{[9]}$. With the development of Internet technology and the popularity of the Internet, the network has gradually penetrated into every corner of life Internet directly affect our lives, the information network is growing into a "fourth media", as people access to information and achieve a variety of social functions The main carrier. The number of Internet users in China ranks first in the world, people's daily life more and more inseparable from the Internet. Therefore, the use of the Internet to build a focus on domestic and foreign anorectal society elite exchange of academic platform, the establishment of a cohesive professional team, both conducive to the healthy development of anorectal discipline, but also for the majority of patients with anorectal disease to provide medical counseling services for the construction of harmony Doctor-patient relationship to contribute.

\section{Conclusions}

Online medical information resources professional consulting platform as a way to obtain new information, has just started construction in the country, its development speed will be extremely rapid. It attracts more and more patients with its convenient, fast and informative features. However, the current professional anorectal site less, less information on the site, update the speed of poor quality of academic exchanges, doctors and students exchange platform is not perfect. Therefore, by the Chinese Association of Higher Education Clinical Education Research Association Anorectal Branch, China Association of constipation, Chengdu anorectal specialist hospital contractor in China an anorectal network has been successfully registered in Chengdu. This site aims to integrate the national anorectal network resources, services for the majority of patients with anorectal disease. For the majority of patients to provide authoritative, comprehensive, free information and information; for the rectal surgeon to build the latest information sharing and exchange platform.

\section{References}

[1] Bhatia Y, Singh S, Rattan KN, Parmar P, Sahni D, Sen R. Anorectal Malformations: Histomorphological and Immunohistochemical Evaluation of Neuronal Dysfunction. J Neonatal Surg. 2017. 6(2): 29. 
[2] Cavina E, Seccia M, Banti P, Zocco G. Anorectal reconstruction after abdominoperineal resection. Experience with double-wrap graciloplasty supported by low-frequency electrostimulation. Dis Colon Rectum. 1998. 41(8): 1010-6.

[3] Bonsang B, Lesourd A, Gavard J, Doucet L, Grasset D, Marcorelles P. [An anorectal lesion: An unexpected association!]. Ann Pathol. 2015. 35(5): 456-9.

[4] Vermes G, László D, Czeizel AE, Ács N. Birth outcomes of patients with isolated anorectal malformations: A population-based case-control study. Congenit Anom (Kyoto). 2016. 56(1): 41-5.

[5] Pandey V, Gangopadhyay AN, Gupta DK, Sharma SP, Kumar V. Management of anorectal malformation without ligation of fistula: an approach preventing posterior urethral diverticula. $\mathbf{J}$ Pediatr Urol. 2014. 10(6): 1149-52.

[6] Qazi SH, Faruque AV, Mateen KMA, Saleem U. Functional Outcome of Anorectal Malformations and Associated Anomalies in Era of Krickenbeck Classification. J Coll Physicians Surg Pak. 2016. 26(3): 204-7.

[7] Chon HK, Shin IS, Kim SW, Lee ST. High grade anorectal stricture complicating Crohn's disease: endoscopic treatment using insulated-tip knife. Intest Res. 2016. 14(3): 285-8.

[8] Falch C, Mueller S, Kirschniak A, Braun M, Koenigsrainer A, Klumpp B. Anorectal malignant melanoma: curative abdominoperineal resection: patient selection with 18F-FDG-PET/CT. World J Surg Oncol. 2016. 14(1): 185.

[9] Liu LG, Yan XB, Shan ZZ, et al. Anorectal functional outcome following laparoscopic low anterior resection for rectal cancer. Mol Clin Oncol. 2017. 6(4): 613-621. 